\title{
Verzeichnis der verwendeten Siglen und Abkürzungen
}

\author{
Siglen
}

Amphilochius von Iconium, Epistula synodalis

La Florenz, Biblioteca Medicaea Laurenziana, Plut. IV I 2 s. XV

Ps Paris, Bibliothèque nationale de France, Supplément grec io2o s. XI

Athanasius von Alexandrien, Epistula ad Alexandrinos

$\mathrm{P}$

Berlin, Staatliche Museen - Preußischer Kulturbesitz, Ägypti- $\quad$ s. V/VI sches Museum und Papyrussammlung, P. I I948

Athanasius von Alexandrien, Epistula ad Jovianum

a Übereinstimmung der Handschriften B K P O R E

B Basel, Universitätsbibliothek, Ms. A III $4 \quad$ s. XIII

E San Lorenzo el Escorial, Real Biblioteca, Ms. S.III.I 5 s. XIII

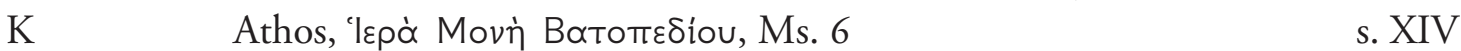

O San Lorenzo el Escorial, Real Biblioteca, Ms. X.II.I I s. XIII-XIV

P Parma, Biblioteca Palatina, Palat. Io s. XIII

R Paris, Bibliothèque nationale de France, Grec 474 s. XI

D Mailand, Biblioteca Ambrosiana, Ms. D 5 I sup. s. XIV

Thdt. Überlieferung bei Theodoret

Tim. Beryt. Überlieferung bei Timotheus von Berytus

Athanasius von Alexandrien, Petitiones Arianorum

B Basel, Universitätsbibliothek, Ms. A III 4 s. XIII

E San Lorenzo el Escorial, Real Biblioteca, Ms. $\Omega$.III.I $5 \quad$ s. XIII

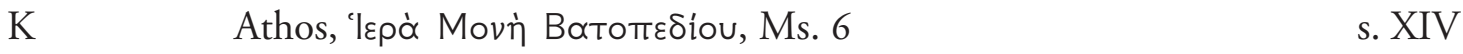

O San Lorenzo el Escorial, Real Biblioteca, Ms. X.II.I I s. XIII/XIV

R Paris, Bibliothèque nationale de France, Grec 474 s. XI

Basilius von Caesarea, Epistulae

A Paris, Bibliothèque nationale de France, Bibliothèque de l'Arse- $\quad$ s. XI nal, Ms. 234 
Paris, Bibliothèque nationale de France, Supplément grec I020

\section{Codex Veronensis LX}

V Verona, Biblioteca Capitolare, Ms. LX

\section{Codex Theodosianus}

\section{CJ Codex Justinianus}

coll. Colb. collectio Colbertina

coll. Quesn. collectio Quesnelliana

$\mathrm{D}$

E

L

$\mathrm{O}$

$\mathrm{V}$

W

Y

Paris, Bibliothèque nationale de France, Latin I 2445

Ivrea, Biblioteca capitolare, Ms. 35

Paris, Bibliothèque nationale de France, Latin 4403

Oxford, Bodleian Library, MS. Arch. Selden. B. I6

Vatikanstadt, Biblioteca Apostolica Vaticana, Reg. lat. 886

Turin, Biblioteca Nazionale Universitaria A. II. 2 (deperditus); Vatikanstadt, Biblioteca Apostolica Vaticana, Vat. lat. 5766

Berlin, Staatsbibliothek - Preußischer Kulturbesitz, Ms. Phill. I74I

\section{Doctrina patrum}
A
B
Vatikanstadt, Biblioteca Apostolica Vaticana, Vat. gr. 2200

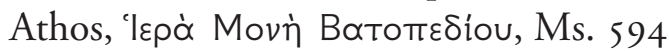
C
Oxford, Bodleian Library, Auct. T. . 6 (Misc. I 84)
Paris, Bibliothèque nationale de France, Grec i I 44
Vatikanstadt, Biblioteca Apostolica Vaticana, Vat. gr. I Io2

s. VIII/IX

s. XII

s. XII (I.V.)

s. XV

Epiphanius von Salamis, Panarion 
Eugenius, Expositio ad Athanasium

Eunomius von Cyzicus, Apologia

C
München, Bayerische Staatsbibliothek, Cod. graec. 5 I 2

s. XV

Paris, Bibliothèque nationale de France, Grec 965

s. XI

Wolfenbüttel, Herzog August Bibliothek, Cod. Guelf. 85 Gud.

s. XIV graec.

Athos, Movì 'I

s.XIV?

\section{Eutyches, Florilegium testimonium patrum}

Novara, Biblioteca Capitolare, cod. XXX

s. IX/X

Gregor von Nyssa, Epistulae

Vatikanstadt, Biblioteca Apostolica Vaticana, Barb. gr. 29 I

s. XIV/XV

Vatikanstadt, Biblioteca Apostolica Vaticana, Vat. gr. 424

s. XIII/XIV

Berlin, Staatsbibliothek - Preußischer Kulturbesitz, Ms. Phill.

s. IX I 829

Berlin, Staatsbibliothek - Preußischer Kulturbesitz, Ms. Phill.

s. IX I 872

Leiden, Universitaire Bibliotheken, Voss. lat. Q. I I

s. IX

Leiden/Paris/Vatikanstadt, Codex Floriacensis fragm.

s. V

\section{Hilarius von Poitiers, Contra Auxentium}

Übereinstimmung der Handschriften C L o

Paris, Bibliothèque nationale de France, Latin 9807

s. V

London, British Museum, Harley 3 I I 5

s. IX

Brüssel, KBR, ms. II 256I

s. IX

Übereinstimmung der Handschriften K F

Paris, Bibliothèque nationale de France, Latin I 695

s. XI

Cambrai, Bibliothèque municipal, 436 (olim 409)

s. IX

Vatikanstadt, Biblioteca Apostolica Vaticana, Arch. Cap. S. Pie-

s. VI tro $\mathrm{D}$ I 82 
Hilarius von Poitiers, Collectanea antiariana

A Paris, Bibliothèque nationale de France, Bibliothèque de l'Arsenal, Ms. 483

\section{Historia Athanasii}

Verona, Biblioteca Capitolare, Ms. LX

Pamphilus, Solutio

\section{Ps.-Athanasius, Epistula catholica}

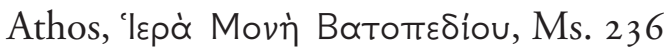

Vatikanstadt, Biblioteca Apostolica Vaticana, Vat. gr. 668

Philostorgius, Historia ecclesiastica

\section{Ps.-Athanasius, Refutatio hypocriseos}

Venedig, Biblioteca Marciana, Ms. gr. Z. 502

Mailand, Biblioteca Ambrosiana, Ms. I 59 sup.

Basel, Universitätsbibliothek, Ms. A III 4, f. I 86a-b

Basel, Universitätsbibliothek, Ms. A III 4, f. 299b-30oa

Florenz, Biblioteca Medicaea Laurenziana, San Marco 695

Florenz, Biblioteca Medicaea Laurenziana, Plut. IV 20

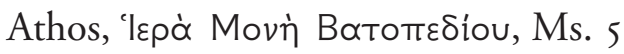

London,British Library, Burney gr. 46

Venedig, Biblioteca Marciana, Ms. gr. Z 49

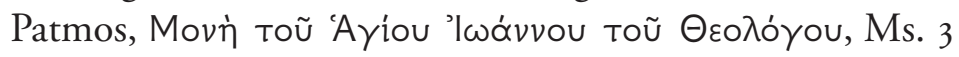

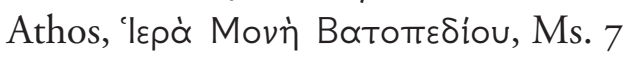

Moskau, Государствеииый Исторический Музей, Sinod gr. s. IX

$$
\text { s. VII }
$$

s. XII/XIII

s. XIV

s.XIV

s. XIII

s. XIII

s. XIII

s. XI/XII

s. XII/XIII

s.XI

s. XI/XII

s. XIII/XIV

s. XIII

s. XIII

s. XIII

s. XIV

s. XII

s. XIV

s. XII

s. XII/XIII

s. XI

s. XI/XII

s. XV I I 5 (243/CCXXX) 
Rufin von Aquileia, Historia ecclesiastica

$\begin{array}{llr}\text { F } & \text { München, Bayerische Staatsbibliothek, Clm 6375 } & \text { s. IX/X } \\ \text { L } & \text { Lucca, Biblioteca capitolare, Ms. 490 } & \text { a. } 787 \\ \text { N } & \text { Paris, Bibliothèque nationale de France, Latin I } 8282 & \text { s. VIII } \\ \text { P } & \text { Vatikanstadt, Biblioteca Apostolica Vaticana, Ms. Palatinus lat. } & \text { s. IX }\end{array}$

822

Socrates, Historia ecclesiastica

A Athos Movì Zвротото́ноu, Ms. 226

s. XIV

Arm. Altarmenische Überlieferung

b Übereinstimmung der Handschriften F M

s. VI/VII

F Florenz, Biblioteca Medicea Laurenziana, Plut. LXIX 5

s. XI

M Florenz, Biblioteca Medicea Laurenziana, Plut. LXX 7

s. X

Cass. lateinische Übersetzung Cassiodors

Sozomenus, Historia ecclesiastica

A Mailand, Biblioteca Ambrosiana, Ms. D 34 sup.

b Übereinstimmung der Handschriften B C

B Oxford, Bodleian Library, Ms. Barocci 142

s. X/XI

C Alexandrien, Bibliothek des Patriarchats 60

s. XIV

Cass. Lateinische Übersetzung Cassiodors

Cod. Nicet. Zitate bei Nicetas

Exc. Trip. Zitate in den Excerpta tripartita

Lib. Synod. Zitate im Libellus Synodi

$\mathrm{V}$

Venedig, Biblioteca Marciana, Ms. gr. Z. 344

s. XIII ex.

Theodoret von Cyrus, Historia ecclesiastica

B Oxford, Bodleian Library, Auct. E IV I8 (misc. 6I)

s. XII/XIII

Cass. lateinische Übersetzung bei Cassiodor

r Übereinstimmung der Handschriften $\mathrm{n} s$

n Übereinstimmung der Handschriften $\mathrm{H} \mathrm{N}$

$\mathrm{H} \quad$ Paris, Bibliothèque nationale de France, Grec I442

s. XIII

$\mathrm{N}$

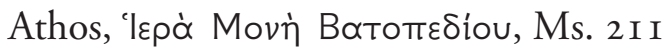

s. XIII

Niceph. $\quad$ Überlieferung bei Nicephorus

s Übereinstimmung der Handschriften G P S

G Rom, Bibliotheca Angelica, Ms. gr. 4I

s. XII/XIII

P Sankt-Peterburg, Российская Национальная Библиотека, $\phi$

s. X

No.906 (gr.)7 I 5

S San Lorenzo el Escorial, Real Biblioteca, Ms. X.III.I4

s. XII

Venedig, Biblioteca Marciana, Ms. gr. 344

s. XIII

v Übereinstimmung der Handschriften F V V ${ }^{2} \mathrm{R}$ 


\section{Konjektoren ${ }^{2}$}

\begin{tabular}{|c|c|}
\hline Amidon & in Amidon, Philostorgius \\
\hline Bade & bei Durst, Ep. contra Auxentium \\
\hline Batiffol & bei Martin/Albert, Histoire "Acéphale» \\
\hline Bidez & $\begin{array}{l}\text { in Bidez/Winkelmann, Philostorgius Kirchengeschich- } \\
\text { te und Bleckmann/Stein, Philostorgius Kirchenge- } \\
\text { schichte }\end{array}$ \\
\hline Borchart & $\begin{array}{l}\text { bei Bidez/Winkelmann, Philostorgius Kirchengeschich- } \\
\text { te und Bleckmann/Stein, Philostorgius Kirchenge- } \\
\text { schichte }\end{array}$ \\
\hline Camplani & in Camplani, Atanasio e Eusebio \\
\hline Cave & bei Vaggione, Eunomius \\
\hline Chadwick & bei Prestige/Chadwick, Basil and Apollinaris \\
\hline Christopherson & $\begin{array}{l}\text { bei Hansen, Sokrates Kirchengeschichte, Bidez/ } \\
\text { Hansen, Sozomenus Kirchengeschichte und Parmen- } \\
\text { tier/Hansen, Theodoret Kirchengeschichte }\end{array}$ \\
\hline Cod. Paris. gr. I 327 & bei A. v. Stockhausen, Kritische (Neu-)Edition \\
\hline Cod. Paris. lat. I 700 & bei Feder, Hilarii Opera IV \\
\hline Cod. Vallicellianus C 23 & bei Field, On the Communion \\
\hline Cornarius & bei Holl/Dummer, Epiphanius III \\
\hline Cotelier & $\begin{array}{l}\text { bei Riedmatten, La Correspondance und Ecclesiae } \\
\text { Graecae Monumenta II, Paris I68I, 99-104 }\end{array}$ \\
\hline Courtonne & in Courtonne, Basile Lettres \\
\hline Coustant & $\begin{array}{l}\text { bei Feder, Hilarii Opera IV und Durst, Glaubensbe- } \\
\text { kenntnis des Auxentius von Mailand }\end{array}$ \\
\hline Crivelli & $\begin{array}{l}\text { bei Durst, Glaubensbekenntnis des Auxentius von Mai- } \\
\text { land }\end{array}$ \\
\hline Deferrari & bei Riedmatten, La Correspondance \\
\hline Diekamp & bei Fromen, Historia acephala \\
\hline Dindorf & bei Holl/Dummer, Epiphanius III \\
\hline
\end{tabular}

${ }^{2}$ Auflösung der Kurztitel im Literaturverzeichnis ab S. 917 
Durst

Engelbrecht

Erasmus

Erl.

Faber

Fabricius

Feder

Field

Fromen

Garnier/Maran

Gillot

Gothofredus

Hansen

Holl

Holste

Hussey

Karmann

Klostermann

Lefort

Le Quien

Lips

Loofs

Maffei

Martin

Migne

Mingarelli

Montfaucon

Oberthür in Durst, Glaubensbekenntnis des Auxentius von Mailand und Durst, Ep. contra Auxentium

bei Feder, Hilarii Opera IV

bei Durst, Glaubensbekenntnis des Auxentius von Mailand

Arbeitsstelle Athanasius Werke, Erlangen

bei Feder, Hilarii Opera IV

bei Vaggione, Eunomius

in Feder, Hilarii Opera IV

in Field, On the Communion

in Fromen, Historia acephala

bei Riedmatten, La Correspondance (Benedictini)

bei Durst, Glaubensbekenntnis des Auxentius von Mailand

bei Bidez/Winkelmann, Philostorgius Kirchengeschichteund Bleckmann/Stein, Philostorgius Kirchengeschichte

in Hansen, Sokrates Kirchengeschichte, Bidez/Hansen, Sozomenus Kirchengeschichte und Parmentier/ Hansen, Theodoret Kirchengeschichte

in Holl/Dummer, Epiphanius III

bei Schwartz, Sammlung und Field, On the Communion

bei Hansen, Sokrates Kirchengeschichte

in Karmann, Meletius von Antiochien

bei Holl/Dummer, Epiphanius III

bei Camplani, Atanasio e Eusebio

bei Tetz, Markellianer und Athanasios von Alexandrien

bei Durst, Glaubensbekenntnis des Auxentius von Mailand

bei Bidez/Winkelmann, Philostorgius Kirchengeschichte und Tetz, Markellianer und Athanasios von Alexandrien

bei Martin/Albert, Histoire «Acéphale»

in Martin/Albert, Histoire «Acéphale»

bei Tetz, Markellianer und Athanasios von Alexandrien

und Savvidis/Wyrwa, Athanasius Werke I 5

bei Riedmatten, La Correspondance

bei Brennecke/U. Heil/A. v. Stockhausen, Athanasius

Werke II 8, Tetz, Markellianer und Athanasios von Alexandrien und Savvidis/Wyrwa, Athanasius Werke I

5

bei Durst, Glaubensbekenntnis des Auxentius von Mailand 
Opitz

Opitz-Turner

Parmentier

Pasquali

Pieper

Pitou

Rettberg

Schwartz

Sebastiani

Seeck

Stockhausen

Tetz

Valois bei Brennecke/U. Heil/A. v. Stockhausen, Athanasius Werke II 8

in Turner/Opitz, EOMIA II 3 und Martin/Albert, Histoire «Acéphale»

in Parmentier/Hansen, Theodoret Kirchengeschichte in Pasquali, GNO VIII.2

in Pieper, Zwei Blätter

bei Feder, Hilarii Opera IV

bei Tetz, Markellianer und Athanasios von Alexandrien in Schwartz, Sammlung und Schwartz, ACO II 2, I

bei Riedmatten, La Correspondance

bei Martin/Albert, Histoire «Acéphale»

Annette von Stockhausen

in Tetz, Markellianer und Athanasios von Alexandrien bei Hansen, Sokrates Kirchengeschichte, Bidez/ Hansen, Sozomenus Kirchengeschichte, Bidez/ Winkelmann, Philostorgius Kirchengeschichte, Bleckmann/Stein, Philostorgius Kirchengeschichte und Parmentier/Hansen, Theodoret Kirchengeschichte

\section{Autoren und Schriften}

Alterc.

Ambr., ep.

Ambr., fid.

Amm.

Apol., ad Iov.

Apol., fid.

Apol., fr.

Arist., EN

Ath., apol. sec.

Ath., Ar.

Ath., decr.

Ath., ep. Afr.

Ath., ep. Alex.

Ath., ep. Epict.

Ath., ep. Jov.

Ath., ep. Serap.

Ath., h. Ar.

Ath., ind. ep. fest.

Ath., pett. Ar.

Ath., sent.
Altercatio Heraclianii et Germinii

Ambrosius von Mailand, Epistulae

Ambrosius von Mailand, De fide

Ammianus Marcellinus, Res gestae

Apolinaris, Ad Iovianum

Apolinaris, De fide

Apolinaris, Fragmenta

Aristoteles, Nikomachische Ethik

Athanasius von Alexandrien, Apologia secunda

Athanasius von Alexandrien, Contra Arianos

Athanasius von Alexandrien, De decretis

Athanasius von Alexandrien, Epistula ad Afros

Athanasius von Alexandrien, Epistula ad Alexandrinos

Athanasius von Alexandrien, Epistula ad Epictetum

Athanasius von Alexandrien, Epistula ad Jovianum

Athanasius von Alexandrien, Epistulae ad Serapionem

Athanasius von Alexandrien, Historia Arianorum

Athanasius von Alexandrien, Index epistularum festivalium

Athanasius von Alexandrien, Petitiones Arianorum

Athanasius von Alexandrien, De sententia Dionysii 
Ath., syn.

Aug., conl.

Bas., adv. eos

Bas., ep.

Bas., spir.

C. Veron. LX

Cass., hist. trip.

Chron. Ed.

Chron. pasch.

CJ

conc. Ant. c.

conc. Chalc. c.

conc. Const. c.

conc. Nic. c.

conc. Serd. c.

Cons. Const.

CTh

Dam., ep.

Didym., Spir. sanct.

Diog. Laert., vit.

Dion. Al., ref.

Doctr. patr.

Dok.

Epiph., anc.

Epiph., haer.

Eug., exp. Ath.

Eun., apol.

Eun., exp. fid.

Eus., h. e.

Eutr., hist.

Eutych., flor.

Fac., def.

Genn., lib.

Greg. Naz., ep.

Greg. Naz., or.

Greg. Nyss., Abl.

Greg. Nyss., ep.

Greg. Nyss., Eust.
Athanasius von Alexandrien, De synodis

Augustinus, Conlatio cum Maximo

Basilius von Caesarea, Adversus eos qui per calumniam dicunt dici a nobis tres deos

Basilius von Caesarea, Epistulae

Basilius von Caesarea, De spiritu sancto

Codex Veronensis LX

Cassiodor-Epiphanius, Historia ecclesiastica tripartita

Chronicon Edessenum

Chronicon Paschale

Codex Justinianus

Concilium Antiochenum, Canones

Concilium Chalcedonense, Canones

Concilium Constantinopolitanum, Canones

Concilium Nicaenum I, Canones

Concilium Serdicense, Canones

Consularia Constantinopolitana

Codex Theodosianus

Damasus, Epistulae

Didymus von Alexandrien, De spiritu sancto

Diogenes Laertius, Vitae philosophorum

Dionysius von Alexandrien, Refutatio et apologia

Doctrina patrum

Dokumente zum arianischen Streit

Epiphanius von Salamis, Ancoratus

Epiphanius von Salamis, Panarion

Eugenius, Expositio ad Athanasium

Eunomius von Cyzicus, Apologia

Eunomius von Cyzicus, Expositio fidei

Euseb von Caesarea, Historia ecclesiastica

Eutropius, Breviarium Historiae Romanae

Eutyches, Florilegium testimonium patrum

Facundus von Hermiane, Pro defensione trium capitulorum

Gennadius von Marseille, Liber sive diffinitio ecclesiasticorum dogmatum

Gregor von Nazianz, Epistulae

Gregor von Nazianz, Orationes

Gregor von Nyssa, Ad Ablabium quod non sint tres dei

Gregor von Nyssa, Epistulae

Gregor von Nyssa, Ad Eustathium 
Greg. Nyss., Graec.

Greg. Nyss., v. Macr.

Hieron., chron.

Hieron., ep.

Hil., c. Aux.

Hil., c. Const.

Hil., coll. antiar.

Hil., syn.

Hist. Ath.

Joh. Mal., chron

Jos., bell. jud.

Jov., ep. Ath.

Jul., ep.

Jul., or.

Just., Dig.

Just., monoph.

Mar. Vict., adv. Ar.

Marc., frg.

Max., diss.

Michael Syrus, chron.

Niceph., conf.

Or., princ.

Pallad., h. Laus.

Pallad., v. Chrys.

Pamph., solut.

Pass. Art.

Paus., frag. hist.

Philost., h.e.

Plut., mor.

Ps.-Ath., Ar. IV

Ps.-Ath., c. Mac.

Ps.-Ath., ep. cath.

Ps.-Ath., ref.

Ps.-Bas., c. Eun.

Ps.-Ign., Phil.

Ruf., h.e.

Sabinus
Gregor von Nyssa, Ad Graecos

Gregor von Nyssa, Vita Macrinae

Hieronymus, Chronicon

Hieronymus, Epistulae

Hilarius von Poitiers, Contra Auxentium

Hilarius von Poitiers, Contra Constantium

Hilarius von Poitiers, Collectanea antiariana

Hilarius von Poitiers, De synodis

Historia Athanasii

Johannes Malalas, Chronicon

Josephus, De bello judaico

Jovian, Epistula ad Athanasium

Julian, Epistulae

Julian, Orationes

Justinian, Digesta

Justinian, Contra Monophysitas

Marius Victorinus, Adversus Arium

Markell von Ancyra, Fragmenta

Maximini Dissertatio

Michael Syrus, Chronicon

Nicephorus Callistus Xanthopulus, Pseudo-Epiphanii sive Epiphanidis confutatio

Origenes, De principiis

Palladius von Helenopolis, Historia Lausiaca

Palladius von Helenopolis, Dialogus de vita S. Ioannis

Chrysostomi

Pamphilus, Solutio

Passio Artemii

Pausanias, Fragmenta historica

Philostorgius, Historia ecclesiastica

Plutarch, Moralia

Ps.-Athanasius, Contra Arianos IV

Ps.-Athanasius, Contra Macedonianos

Ps.-Athanasius, Epistula catholica

Ps.-Athanasius, Refutatio hypocriseos

Pseudo-Basilius, Contra Eunomium

Pseudo-Ignatius, Epistula ad Philadelphenses

Rufin von Aquileia, Historia ecclesiastica

Sabinus von Heraclea, Synagoge 
Socr., h. e.

Soz., h. e.

Sulp. Sev., chron.

Syn. vet.

Thdr. Lect., epit.

Thdr. Mops., cat.

Thdt., h. e.

Thdt., h. rel.

Theoph., chron.

Urk.

Zos., hist.
Socrates, Historia ecclesiastica

Sozomenus, Historia ecclesiastica

Sulpicius Severus, Chronicon

Synodicon vetus

Theodorus Lector, Epitome

Theodor von Mopsuestia, Katechesen

Theodoret von Cyrus, Historia ecclesiastica

Theodoret von Cyrus, Historia religiosa

Theophanes, Chronographia

Urkunden zum arianischen Streit

Zosimus, Historia nova 
